\title{
PROCESSAMENTO AUDITIVO DE MILITARES EXPOSTOS A RUÍDO OCUPACIONAL
}

\section{Auditory processing of servicemen exposed to occupational noise}

\author{
Carla Cassandra de Souza Santos ${ }^{(1)}$, Luiza de Salles Juchem ${ }^{(2)}$, Angela Garcia Rossi ${ }^{(3)}$
}

\section{RESUMO}

Objetivo: avaliar o processamento auditivo de militares expostos a ruído ocupacional. Métodos: foram avaliados 41 militares, com exposição a ruído superior a 10 anos, subdivididos em Grupo A $(n=16)$, sem perda auditiva e Grupo B $(n=25)$, com perda auditiva. Foram realizadas avaliação audiológica básica e testes de processamento auditivo (testes de Fala Filtrada, SSW em Português e de Padrão de Freqüência). Resultados: observou-se altas incidências de alteração de processamento auditivo, especialmente no teste de Fala Filtrada $(43,75 \%$ e $68 \%$ nos grupos A e B, respectivamente) e teste de Padrão de Freqüência (68,75\% e 48\%, nos grupos A e B, respectivamente). O teste SSW não se mostrou eficiente para avaliar as habilidades auditivas centrais de indivíduos expostos a elevados níveis de pressão sonora. Conclusão: a exposição a ruído ocupacional interfere no processamento auditivo de militares. As alterações na via auditiva central podem ser verificadas independente da presença de alteração auditiva periférica.

DESCRITORES: Audição; Ruído Ocupacional; Testes Auditivos

\section{INTRODUÇÃO}

Atualmente, a perda auditiva induzida por ruído (PAIR) é a segunda maior causa de perda auditiva neurossensorial, logo após a presbiacusia ${ }^{1}$. Tal patologia é a enfermidade profissional irreversível de maior ocorrência em todo o mundo ${ }^{2}$.

Há bastante tempo, a literatura audiológica tem mostrado os danos causados à audição pelo ruído intenso. Em 1890, já era feita a descrição de achados anátomo-patológicos detectados na cóclea e no nervo coclear de caldeireiros. Foi verificada a degeneração de células situadas na porção basal da cóclea ${ }^{3}$.

Sabe-se que a PAIR é uma patologia neurossensorial, bilateral e irreversível ${ }^{4-8}$. Várias categorias de profissionais trabalham em locais em que o ruído é suficientemente intenso para causar uma perda

(1) Fonoaudióloga; Militar da Aeronáutica; Mestre em Distúrbios da Comunicação Humana pela Universidade Federal de Santa Maria.

(2) Fonoaudióloga; Integrante da Clínica Escuta em Santa Maria - RS; Mestre em Distúrbios da Comunicação Humana pela Universidade Federal de Santa Maria.

(3) Fonoaudióloga; Professora adjunta da Universidade Federal de Santa Maria; Doutora em Distúrbios da Comunicação Humana pela Universidade Federal de São Paulo. auditiva. Em homens expostos a ruído industrial, por mais de cinco horas por dia por até 35 anos, foram observadas mudanças ocorridas no limiar auditivo na freqüência de $4000 \mathrm{~Hz}$ em função da exposição em anos. Ficou evidente que a máxima mudança ocorreu dos dez aos 12 anos de exposição, independente do nível de ruído ${ }^{9}$.

Em mulheres tecelãs expostas a ruído intenso, foi verificada perda da acuidade auditiva nos primeiros dez a 15 anos de exposição, seguido por um período de cerca de dez anos na qual a deterioração foi muito pequena ${ }^{10}$.

De 300 funcionários de uma usina siderúrgica e metalúrgica, foram encontradas $34 \%$ de audiometrias alteradas. Além disso, muitas queixas auditivas e neuro-vegetativas foram citadas pelos indivíduos, como, por exemplo, $18,3 \%$ deles relataram diminuição da acuidade auditiva para conversação. Todas as queixas foram mais freqüentes em indivíduos com audiometria alterada e no grupo de trabaIhadores com dois a cinco anos de serviço ${ }^{11}$.

Metalúrgicos também foram alvos de outro estudo, sendo todos os avaliados com idade entre 56 e 68 anos e mais de 30 anos de exposição a ruído intenso. Os resultados mostraram que 52\% dos indivíduos até 62 anos e $67 \%$ dos indivíduos com mais de 62 anos apresentaram perda audi- 
tiva severa nas freqüências de 3,4 ou $6 \mathrm{KHz}$. Os testes de discriminação vocal com o uso de monossílabos no silêncio foram encontrados dentro do padrão de normalidade em quase 100\% da amostra. Os mesmos testes aplicados com ruído causaram uma diminuição dos índices com piora de cerca de $20 \%{ }^{12}$. As alterações auditivas sugestivas de PAIR em metalúrgicos jovens avaliados por outros autores, totalizaram $21 \%$ e $41 \%$ dos avaliados, respectivamente ${ }^{13,14}$.

Além da perda auditiva, as pessoas expostas a ruído ocupacional costumam relatar diversas queixas. Sensação de perda auditiva, dificuldade de comunicação, zumbido, intolerância a sons intensos foram algumas queixas encontradas na literatura ${ }^{15}$. Neste estudo, a sensação de perda auditiva mostrou uma nítida progressão, de acordo com o tempo de exposição a ruído. Houve um aumento considerável na dificuldade de comunicação em indivíduos que estavam trabalhando expostos a ruído há mais de 12 anos.

Muitas queixas auditivas, além de um percentual de $50 \%$ de alterações auditivas à audiometria, foram encontradas em funcionários de seções de manutenção e lavanderia de um hospital ${ }^{16}$. Em ex-funcionários de uma indústria de bebidas, onde todos os avaliados já possuíam perda auditiva neurossensorial, as queixas mais relatadas foram: zumbido $(68,7 \%)$, disacusia $(62,5 \%)$, recrutamento $(56,2 \%)$ e dificuldade de compreensão de fala $(43,7 \%)^{17}$.

Os militares constituem o grupo estudado por diversos autores e sabe-se que esses profissionais têm alta incidência de perda auditiva ${ }^{18-20}$. Pesquisadores avaliaram os perfis auditivos de um grupo de militares de uma unidade do exército. Foram avaliados 99 sujeitos do sexo masculino, nos quais verificou-se alteração em $38,1 \%$ dos traçados audiométricos, sendo a maioria sugestiva de PAIR. A perda auditiva encontrada foi mais intensa quanto maior a idade e o tempo de serviço ${ }^{20}$.

Os indivíduos que se expõem ao ruído em seus ofícios, periodicamente devem ser submetidos à avaliação auditiva. De acordo com NR-7, do Ministério do Trabalho e Emprego ${ }^{21}$, o trabalhador deve ser submetido à audiometria tonal liminar por via aérea e, em caso de alterações, avaliação por via óssea e determinação dos limiares de reconhecimento de fala. Com este tipo de avaliação, facilmente, é possível verificar a presença ou não de PAIR. Esse déficit sensorial tem sido bastante estudado e, nesse caso, o dano auditivo tem sua localização na porção coclear do sistema auditivo ${ }^{3,6}$.

Porém, sabe-se que a audição não é apenas a percepção da presença dos sons. Além disso, a audiometria tonal é limitada para informar sobre a capacidade de comunicação dos indivíduos, pois a percepção da fala envolve a audição periférica e a central ${ }^{22}$.

Processamento auditivo é o conjunto de habilidades específicas que permitem o indivíduo interpretar o que ouve. Tal habilidade é mediada por centros auditivos do tronco encefálico e cérebro ${ }^{23}$.

As habilidades auditivas ou os processos do sistema auditivo central são:

- fechamento auditivo - capacidade do ouvinte normal em utilizar redundâncias extrínsecas e intrínsecas para preencher partes distorcidas ou ausentes do som ouvido e reconhecer a mensagem completa;

- separação binaural - capacidade de um ouvinte em processar a mensagem auditiva captada por uma orelha, enquanto ignora outra mensagem, apresentada simultaneamente a outra orelha;

- integração binaural - habilidade de processar informações distintas que são apresentadas ao mesmo tempo às duas orelhas;

- padrão temporal-habilidade de reconhecimento de contornos acústicos de um sinal;

- interação binaural - localização e lateralização dos estímulos auditivos, detecção de sinais na presença de ruído e fusão binaural ${ }^{24}$.

$\mathrm{Na}$ literatura, encontram-se vários testes para a avaliação da função auditiva central. Na década de 1950, foi elaborado um teste para avaliar pacientes com tumores de lobo temporal. A técnica empregada foi a de apresentar listas de palavras distorcidas por um filtro passa-baixo. Na grande maioria dos casos avaliados, a discriminação foi muito pior na orelha oposta ao lado da lesão cerebral. Nos mesmos sujeitos, a audiometria tonal e vocal tradicionais falharam, não revelando a anormalidade ${ }^{25}$.

Para a adequada avaliação do processamento auditivo, deve ser feita uma bateria de testes que inclua um teste de baixa redundância, um dicótico e um de padrão temporal. Dessa forma, as funções auditivas centrais comprometidas podem ser apontadas ${ }^{26}$.

O teste de fala filtrada tem baixa redundância e é sensível para identificar disfunções associadas ao sistema nervoso central podendo ser útil em identificar a presença de lesão. É fidedigno para medir a habilidade de fechamento auditivo ${ }^{27}$.

O teste Dicótico de Dissílabos Alternados ou SSW (sigla para Staggered Spondaic Word Test) foi criado em $1962{ }^{28}$ nos Estados Unidos. No Brasil, após a realização de sua versão para o português ${ }^{29}$, tem sido empregado em diversas pesquisas, pois avalia as habilidades auditivas de memória para sons em seqüência e figura-fundo para sons verbais ${ }^{29}$.

O SSW tem características importantes, que justifica o seu uso: não sofre interferências de perdas 
auditivas periféricas, é simples e fácil de aplicar, tem padronização de seus resultados na faixa de cinco a 70 anos de idade, é confiável, válido e de fácil aplicação ${ }^{30}$.

A literatura mostra estudos importantes em que houve a aplicação do teste SSW para avaliar idosos ${ }^{31}$; pacientes com traumatismo cranioencefálico e acidentes vasculares cerebrais ${ }^{32}$; indivíduos com perda auditiva neurossensorial, com e sem exposição a ruído ${ }^{33}$; trabalhadores com exposição a ruído ocupacional ${ }^{34}$ e também com PAIR já instalada ${ }^{35}$; além de exposição simultânea a ruído e outras substâncias encontradas em ambiente ocupacional 2,36 .

O teste de padrão de freqüência (PPS) é um teste de padrão temporal. Na habilidade de ordenar e seqüencializar os estímulos acústicos são necessárias várias habilidades, dentre elas, as de reconhecer o estímulo acústico isoladamente, discriminá-lo em relação a outros estímulos, armazená-lo por um curto período de tempo e reproduzi-lo ${ }^{37}$. Pacientes com disfunção auditiva cortical, tanto em hemisfério direito quanto em esquerdo, mostram um prejuízo nas habilidades de realizar as tarefas propostas em tal teste, tanto na forma murmurada quanto na nomeada ${ }^{38}$. Este teste é sensível para detectar diferentes lesões cerebrais; sendo menos sensível para detectar lesões de tronco cerebral ${ }^{39}$.

Testes não-verbais devem ser aplicados, pois nenhum teste de fala, sozinho é sensível para todas as patologias e disfunções do sistema nervoso auditivo central ${ }^{40}$. Além disso, testes de fala podem mascarar dificuldades importantes de processamento auditivo, uma vez que o ouvinte pode usar habilidades lingüísticas e intelectuais para compensar a dificuldade de processamento ${ }^{41}$.

As baterias de testes, entretanto, não precisam, necessariamente, ser extensas, pois dois ou três testes selecionados apropriadamente podem ser suficientes. A ênfase dada à avaliação auditiva central é devida ao fato de que o sistema nervoso auditivo central pode ser afetado secundariamente por outros distúrbios ${ }^{42}$.

As alterações de processamento auditivo não são verificadas pelos dados obtidos através da avaliação audiológica convencional em ambiente ocupacional ${ }^{34}$. Freqüentemente, pessoas com PAIR reclamam de dificuldade em reconhecer a fala em situações de escuta desfavorável, como por exemplo, em ambientes ruidosos ${ }^{43}$. O autor aplicou nove listas de palavras monossilábicas, com 25 palavras cada uma. As listas foram apresentadas a 30 voluntários otologicamente normais, a oito indivíduos de audição normal e a 65 trabalhadores expostos a ruído ocupacional. Os resultados do teste em trabalhadores expostos a ruído ocupacional não mostraram diferenças significativas em relação a sujeitos normo-ouvintes, quando o exame foi feito sem o ruído; porém, diferenças significativas ocorreram quando os testes foram realizados com o ruído. Isso mostra que, os testes de fala, com ruído competidor, são capazes de apontar desabilidades auditivas em portadores de PAIR ${ }^{43}$.

Há poucos estudos enfocando as alterações da função auditiva central de pessoas expostas a ruído ocupacional. Em uma pesquisa do processamento auditivo em militares, com e sem perda de audição, expostos a elevados níveis de pressão sonora, os testes SSW, PPS, e Fala Filtrada foram utilizados. Foi concluído que, independente da presença de alterações periféricas da audição, a exposição a ruído intenso tem efeitos deletérios no processamento auditivo de militares ${ }^{34}$.

Em outro estudo, o reconhecimento de fala de portadores de PAIR foi avaliado. Além do Índice Percentual de Reconhecimento de Fala (IPRF), o teste SSW e teste de Fala com Ruído foram aplicados. Os autores concluíram que o IPRF e o SSW são nada ou pouco úteis para discriminar portadores de PAIR de normo-ouvintes e o teste de Fala com Ruído pode ser sensível para identificar alterações auditivas em pacientes com PAIR ${ }^{35}$. Em operadores de telemarketing, o processamento auditivo foi avaliado por meio dos testes de Fala Filtrada, Random Gap Detection Test (RGDT) e Masking Level Difference (MLD). Foram observadas $45 \%$ de alterações no RGDT e $25 \%$ no MLD ${ }^{44}$.

Além do dano coclear, muitas pesquisas sugerem que há uma perda funcional no sistema nervoso auditivo central por exposição a ruído intenso ${ }^{45,46}$. Cabe ser lembrado que, além da exposição a ruído, outros riscos, tais como o chumbo ${ }^{2}$, tolueno ${ }^{47} \mathrm{e}$ várias espécies de solventes ${ }^{36,48}$ podem ser causadores de alterações nas vias auditivas centrais.

Assim, o objetivo deste estudo foi o de avaliar o processamento auditivo de militares expostos a ruído ocupacional.

\section{MÉTODOS}

Militares da Aeronáutica ficam expostos a ruído intenso em suas atividades diárias, como, por exemplo, ao elevado ruído do disparo de armas de fogo, de helicópteros, de aviões, entre outros. Os militares avaliados pertencem ao Esquadrão do Suprimento e Manutenção (ESM), local destinado a dar apoio à aviação, fazendo a revisão e a manutenção das aeronaves. Independente de haver movimentação de aeronaves na pista (pouso e decolagem), o ESM continua ruidoso, pois neste espaço é feito o teste de motores, testes hidráulicos, uso de ferramentas 
pneumáticas, além de outras atividades que envolvem ruído ocupacional.

Os participantes deste estudo são todos destros, do sexo masculino, com tempo de serviço superior a dez anos. O grupo estudado foi composto de 41 militares sendo divididos em dois grupos: grupo A, composto por indivíduos sem perda auditiva $(\mathrm{N}=16$, $39 \%$ ) e grupo $B$, composto por militares com perda auditiva ( $\mathrm{N}=25,61 \%)$.

A audição foi considerada normal quando todos os limiares de audibilidade forem de até $25 \mathrm{~dB}$ NA e houve perda auditiva quando pelo menos um dos limiares for superior a $25 \mathrm{~dB} N{ }^{49}$.

Todos os 41 indivíduos que aceitaram participar da presente pesquisa se enquadraram nos critérios de inclusão propostos:

- não apresentar comprometimento na orelha externa e/ou orelha média;

- apresentar timpanograma Tipo A 50;

- possuir limiares auditivos até $40 \mathrm{~dB}$ NA nas freqüências de 500, 1000 e $2000 \mathrm{~Hz}$, acuidade auditiva simétrica entre as orelhas e que o IPRF para monossílabos fosse no mínimo de $70 \%$ em cada orelha ${ }^{51}$.

Após assinarem o Termo de Consentimento Livre e Esclarecido, que explicou os procedimentos realizados neste estudo, os indivíduos foram submetidos aos seguintes procedimentos, além de anamnese: avaliação com médico otorrinolaringologista, a avaliação audiológica básica, composta de audiometria tonal liminar; Limiar de Reconhecimento de Fala (LRF); Índice Percentual de Reconhecimento de Fala (IPRF) e medidas de imitância acústica. Previamente à avaliação, foi exigido repouso acústico de 14 horas $^{6}$.

A avaliação da função auditiva central foi verificada por meio da aplicação dos testes de Fala Filtrada, teste Dicótico de Dissílabos Alternados (tradução em português para SSW - Staggered Spondaic Word) e teste do Padrão de Freqüência (PPS).

O teste de fala filtrada foi aplicado a $50 \mathrm{~dB} N{ }^{27}$. A avaliação foi feita utilizando-se os 25 monossílabos com significado, distorcidos por filtro passabaixo ${ }^{52,53}$. Iniciou-se pela orelha direita e, logo após, outra seqüência foi apresentada à orelha esquerda. A resposta solicitada é a repetição oral da palavra ouvida. Os índices percentuais de reconhecimento de Fala Filtrada abaixo de $70 \%$ foram considerados anormais ${ }^{27}$.

O teste SSW adaptado para o português ${ }^{29}$ constou de 40 seqüências de quatro palavras dissilábicas, apresentadas em quatro condições, duas sem competição (direita não-competitiva - DNC e esquerda não-competitiva - ENC) e duas com competição (direita competitiva - DC e esquerda competitiva - EC). A aplicação foi feita a 50 dB NS e o paciente deveria repetir as palavras na ordem ouvida ${ }^{29}$. A análise dos resultados foi quantitativa, ou seja, através do cálculo da porcentagem de erros para cada condição das orelhas separadamente e a porcentagem do total de erros no teste SSW. Considerou-se normal acertos iguais ou superiores a $90 \%$, tanto nas quatro condições de pesquisa (DNC, DC, EC, ENC), quanto no total de acertos ${ }^{51}$.

Em relação ao teste de padrão de freqüência (PPS), foi utilizado o da Auditec ${ }^{54}$ e aplicado de forma monoaural em um nível de intensidade de 50 dB NS. Seqüências de três tons foram apresentadas com duração de $500 \mathrm{~ms}$, havendo tons altos $(1430 \mathrm{~Hz})$ e baixos $(880 \mathrm{~Hz})$. A elevação e a queda dos tons foram de $10 \mathrm{~ms}$. O intervalo entre os três tons foi de $300 \mathrm{~ms}$ e o intervalo entre cada seqüência de $10 \mathrm{~s}$. Testou-se primeiro a orelha direita e, logo depois, a orelha esquerda. Apresentaram-se 60 seqüências de tons para cada orelha, sendo as 30 primeiras com resposta solicitada do tipo murmúrio (imitação) e as demais com respostas verbais, havendo a nomeação da seqüência ouvida (usouse os termos "fino" e "grosso" para referir-se às freqüências alta e baixa, respectivamente). O padrão de normalidade considerado foram percentuais de acertos superiores a $76 \%{ }^{55}$.

Para realização desta pesquisa, utilizaram-se os seguintes materiais: Audiômetro GSI 61 com fones TDH - 39 e coxim MX - 41; analisador de orelha média Interacoustics AZ 7, com fone TDH-39 e coxim MX-41, com tom-sonda de $220 \mathrm{~Hz}$; CD-Player da marca Britânia, modelo BS 279, acoplado ao audiômetro; CDs com as gravações dos testes de Fala Filtrada, SSW e Padrão de Freqüência (PPS).

O projeto do presente estudo foi aprovado pelo Comitê de Ética em Pesquisa da Universidade Federal de Santa Maria (CEP - UFSM), sob número 23081005283/2006-22 e CAAE 00220243000-06.

Em relação à análise estatística, considerandose a não-normalidade dos dados, aplicou-se o teste não-paramétrico o teste de Kruskal-Wallis, para a comparação entre os dois grupos avaliados ( $A$ e B $)$ nesta pesquisa. $O$ nível de significância utilizado foi de $5 \%(p<0,05)$.

\section{RESULTADOS}

A idade dos 41 participantes deste estudo, dos grupos $A(N=16)$ e $B(N=25)$, variou de 31 a 51 anos (Figura 1).

O tempo de serviço com exposição a ruído intenso dos participantes dos grupos A e B variou de 12 a 31 anos (Figura 1). 


\begin{tabular}{|c|c|c|c|c|}
\hline \multirow{2}{*}{} & \multicolumn{2}{|c|}{ IDADE } & \multicolumn{2}{c|}{ TEMPO DE SERVIÇO } \\
\cline { 2 - 5 } & Grupo A & Grupo B & Grupo A & 12,00 \\
\hline Mínimo & 31,00 & 31,00 & 31,00 & 31,00 \\
Máximo & 47,00 & 51,00 & 22,19 & 24,40 \\
Média & 40,44 & 42,48 & 5,419 & 5,091 \\
Desvio Padrão & 5,059 & 5,324 & \multicolumn{2}{c|}{0,1757} \\
\hline P & \multicolumn{2}{|c|}{0,1760} \\
\hline
\end{tabular}

Não existe diferença significante entre as médias $(p>0,05)$ - Teste de Kruskal-Wallis.

Figura 1 - Idades e tempo de serviço dos indivíduos dos grupos A e B, em anos

Em relação à idade e ao tempo de serviço dos militares, não se verificou diferença estatisticamente significante entre os grupos A e B.

Conforme já especificado nos métodos desta pesquisa, a avaliação audiológica básica detectou $39 \%$ de indivíduos com a audição normal e $61 \%$ de militares com perda auditiva. As medidas de imitância acústica serviram apenas para cumprir os critérios de inclusão de militares na presente pesquisa.

Na Tabela 1, observam-se os resultados do teste de Fala Filtrada em percentuais para os indivíduos dos dois grupos estudados, em função das orelhas direita e esquerda.

Tabela 1 - Percentuais de acertos no teste de Fala Filtrada dos indivíduos dos grupos A e B, em função das orelhas direita e esquerda

\begin{tabular}{lcccc}
\hline & \multicolumn{3}{c}{ TESTE DE FALA FILTRADA } \\
\hline & \multicolumn{2}{c}{ GRUPO A } & \multicolumn{3}{c}{ GRUPO B } \\
\cline { 2 - 6 } & Direita & Esquerda & Direita & Esquerda \\
Mínimo & 60,00 & 52,00 & 44,00 & 40,00 \\
Máximo & 84,00 & 88,00 & 80,00 & 88,00 \\
Média & 72,50 & 74,75 & 64,32 & 71,20 \\
Desvio Padrão & 7,85 & 9,43 & 11,25 & 12,05 \\
\hline P & \multicolumn{2}{c}{0,3595} & \multicolumn{3}{c}{$0,0266^{*}$} \\
\hline
\end{tabular}

* Existe diferença estatisticamente significante entre os resultados do teste de Fala Filtrada do grupo B, entre as orelhas direita $\mathrm{e}$ esquerda - Teste de Kruskal-Wallis.

Pode-se observar na Tabela 1 que não houve diferença estatisticamente significante entre as orelhas direita e esquerda, nos resultados obtidos pelos indivíduos do grupo A no teste de Fala Filtrada. Na mesma comparação, feita com o grupo $B$, foi evidenciada diferença estatisticamente significante entre as orelhas direita e esquerda.
A Tabela 2 mostra os resultados obtidos no teste de Fala Filtrada, verificados em ambos as orelhas dos indivíduos em função dos grupos A e B.

Ao se comparar os resultados do teste de Fala Filtrada entre os grupos A e B, não foi verificada diferença estatisticamente significante entre os valores obtidos na orelha esquerda. Porém, entre

Tabela 2 - Percentuais de acertos no teste de Fala Filtrada das orelhas direita e esquerda dos indivíduos, em função dos grupos A e B

\begin{tabular}{lcccc}
\hline & \multicolumn{4}{c}{ TESTE DE FALA FILTRADA } \\
\cline { 2 - 5 } & \multicolumn{2}{c}{ Orelha Direita } & Orelha Esquerda \\
\cline { 2 - 5 } & $\mathrm{A}$ & $\mathrm{B}$ & $\mathrm{A}$ & $\mathrm{B}$ \\
Mínimo & 60,00 & 44,00 & 52,00 & 40,00 \\
Máximo & 84,00 & 80,00 & 88,00 & 88,00 \\
Média & 72,50 & 64,32 & 74,75 & 71,20 \\
Desvio Padrão & 7,85 & 11,25 & 9,43 & 12,05 \\
\hline
\end{tabular}

* Existe diferença estatisticamente significante entre os resultados do teste de Fala Filtrada dos grupos A e B, apenas para a orelha direita - Teste de Kruskal-Wallis. 
os valores médios do teste de Fala Filtrada dos grupos A e B, foi evidenciada diferença estatisticamente significante para a orelha direita.

$\mathrm{Na}$ Figura 2, verifica-se a quantidade de alterações no teste de Fala Filtrada em função do número de indivíduos para os grupos A e B.

No teste de Fala Filtrada, $43,75 \%$ e $68 \%$ dos indivíduos do grupo A e B, respectivamente, tiveram resultados abaixo de $70 \%$ em pelo menos uma orelha.

Em relação ao teste SSW, a Tabela 3 apresenta o número de erros que os avaliados obtiveram nas quatro condições avaliadas: direita não competitiva (DNC), direita competitiva (DC), esquerda competitiva (EC) e esquerda não competitiva (ENC); além de apresentar o total de erros.

Conforme se verifica na Tabela 3, ao se comparar os resultados do teste SSW nas quatro condições pesquisadas e quanto ao total de erros, não houve diferença estatisticamente significante entre os grupos A e B.

Na Figura 3 estão expostos os números de erros e as respectivas porcentagens obtidas no teste SSW, para os grupos A e B.

\begin{tabular}{|c|c|c|c|c|}
\hline \multirow{2}{*}{} & \multicolumn{4}{|c|}{ ALTERAÇÕES NO TESTE DE FALA FILTRADA } \\
\cline { 2 - 5 } & \multicolumn{2}{|c|}{ GRUPO A } & N & $\%$ \\
\cline { 2 - 5 } & $\mathrm{N}$ & 18,75 & 8 & 32,00 \\
\hline Orelha Direita & 3 & 6,25 & 0 & 0,00 \\
Orelha Esquerda & 1 & 18,75 & 9 & 36,00 \\
Orelha Direita + & 3 & 56,25 & 8 & 32,00 \\
Orelha Esquerda & 9 & 100,00 & 25 & 100,00 \\
Sem alteração & 16 & & \\
\hline Total & &
\end{tabular}

Figura 2 - Alterações encontradas no teste de Fala Filtrada em função do número de indivíduos para os grupos A e B

Tabela 3 - Resultados obtidos (erros) nas quatro condições e no total de erros no teste SSW

\begin{tabular}{|c|c|c|c|c|c|c|c|c|c|c|}
\hline \multicolumn{11}{|c|}{ TESTE SSW } \\
\hline & \multicolumn{2}{|c|}{ DNC } & \multicolumn{2}{|c|}{$\mathrm{DC}$} & \multicolumn{2}{|c|}{ EC } & \multicolumn{2}{|c|}{ ENC } & \multicolumn{2}{|c|}{ Total } \\
\hline & $A$ & $B$ & $A$ & $B$ & $A$ & $B$ & $A$ & $B$ & $A$ & $B$ \\
\hline Mínimo & 0,00 & 0,00 & 0,00 & 0,00 & 0,00 & 0,00 & 0,00 & 0,00 & 0,62 & 0,00 \\
\hline Máximo & 2,00 & 1,00 & 6,00 & 3,00 & 7,00 & 5,00 & 3,00 & 1,00 & 9,37 & 5,00 \\
\hline Média & 0,50 & 0,20 & 1,87 & 1,08 & 2,25 & 1,64 & 0,69 & 0,28 & 3,32 & 1,97 \\
\hline $\begin{array}{l}\text { Desvio } \\
\text { Padrão } \\
\end{array}$ & 0,73 & 0,41 & 1,71 & 1,08 & 2,02 & 1,22 & 0,95 & 0,46 & 2,75 & 1,22 \\
\hline$p$ & \multicolumn{2}{|c|}{0,1668} & \multicolumn{2}{|c|}{0,1369} & \multicolumn{2}{|c|}{0,4633} & \multicolumn{2}{|c|}{0,1763} & \multicolumn{2}{|c|}{0,3452} \\
\hline
\end{tabular}

Não existe diferença significante entre as médias $(p>0,05)$ - Teste de Kruskal-Wallis.

\begin{tabular}{|c|c|c|c|c|}
\hline & \multicolumn{4}{|c|}{ ALTERAÇÕES NO TESTE SSW } \\
\hline & \multicolumn{2}{|c|}{ GRUPO A } & \multicolumn{2}{|c|}{ GRUPO B } \\
\hline & $\mathrm{N}$ & $\%$ & $\mathrm{~N}$ & $\%$ \\
\hline DNC & 0 & 0,00 & 0 & 0,00 \\
\hline DC & 0 & 0,00 & 0 & 0,00 \\
\hline EC & 1 & 6,25 & 1 & 4,00 \\
\hline ENC & 0 & 0,00 & 0 & 0,00 \\
\hline Total de erros & 0 & 0,00 & 0 & 0,00 \\
\hline $\mathrm{DC}+\mathrm{EC}$ & 1 & 6,25 & 0 & 0,00 \\
\hline Sem alteração & 14 & 87,50 & 24 & 96,00 \\
\hline Total & 16 & 100,00 & 25 & 100,00 \\
\hline
\end{tabular}

Figura 3 - Alterações encontradas no teste SSW em função do número de indivíduos, para os grupos A e B 
Tabela 4 - Percentuais de acertos no teste PPS nas orelhas direita e esquerda dos indivíduos dos grupos A e B, em função das respostas murmurada e nomeada

\begin{tabular}{|c|c|c|c|c|c|c|c|c|}
\hline \multicolumn{9}{|c|}{ TESTE PPS } \\
\hline & \multicolumn{4}{|c|}{ GRUPO A } & \multicolumn{4}{|c|}{ GRUPO B } \\
\hline & \multicolumn{2}{|c|}{ Murmurado } & \multicolumn{2}{|c|}{ Nomeado } & \multicolumn{2}{|c|}{ Murmurado } & \multicolumn{2}{|c|}{ Nomeado } \\
\hline & OD & OE & OD & OE & OD & OE & OD & OE \\
\hline Mínimo & 33,30 & 46,60 & 46,60 & 20,00 & 53,30 & 33,30 & 33,30 & 13,30 \\
\hline Máximo & 100,0 & 100,00 & 100,00 & 100,00 & 100,00 & 100,00 & 100,00 & 100,00 \\
\hline Média & 82,90 & 80,40 & 81,20 & 78,30 & 86,00 & 88,00 & 81,20 & 72,80 \\
\hline $\begin{array}{l}\text { Desvio } \\
\text { Padrão }\end{array}$ & 19,78 & 19,10 & 16,55 & 20,86 & 12,32 & 16,90 & 19,22 & 20,63 \\
\hline$p$ & \multicolumn{2}{|c|}{0,4990} & \multicolumn{2}{|c|}{0,8192} & \multicolumn{2}{|c|}{0,2057} & \multicolumn{2}{|c|}{0,0941} \\
\hline
\end{tabular}

Não existe diferença significante entre as médias $(p>0,05)$ - Teste de Kruskal-Wallis.

Em relação ao SSW, $12,5 \%$ e 4\% dos indivíduos do grupo $A$ e $B$, respectivamente, tiveram resultados abaixo de $90 \%$ de acertos em pelo menos uma das seguintes situações: DNC, DC, EC, ENC e total de erros.

$\mathrm{Na}$ Tabela 4 estão apresentados os resultados do teste PPS de ambos os grupos estudados, das orelhas direita e esquerda, nas condições murmurada e nomeada. Fez-se uma comparação por oreIha testada.

Não foi observada diferença estatisticamente significante entre a média dos resultados do teste PPS entre as orelhas esquerda e direita, em nenhuma das quatro situações possíveis: resposta murmurada do grupo $A$ e do grupo $B$ e resposta nomeada do grupo $A$ e do grupo $B$.

Os resultados do teste PPS, em percentuais nas respostas murmurada e nomeada dos indivíduos dos grupos A e B, em função das orelhas direita e esquerda, estão expostos na Tabela 5.

Observa-se que não houve diferença estatisticamente significante entre as respostas dos grupos A e B para cada situação de apresentação do teste PPS, ou seja, murmúrio em orelha direita, nomeação na mesma orelha, murmúrio em orelha esquerda e nomeação na mesma orelha.

Na Figura 4 estão expostos os números de erros e as respectivas porcentagens obtidos no teste PPS dos os grupos A e B.

Em relação ao teste PPS, $68,75 \%$ e $48 \%$ dos indivíduos dos grupos A e B, respectivamente, tiveram resultados abaixo de $76 \%$ em pelo menos uma das condições (murmurada ou nomeada) em pelo menos uma das orelhas.

Tabela 5 - Percentuais de acertos no teste PPS nas respostas murmurada e nomeada dos indivíduos dos grupos A e B, em função das orelhas direita e esquerda

\section{TESTE PPS}

\begin{tabular}{|c|c|c|c|c|c|c|c|c|}
\hline & \multicolumn{4}{|c|}{ Murmurado } & \multicolumn{4}{|c|}{ Nomeado } \\
\hline & \multicolumn{2}{|c|}{ Orelha Direita } & \multicolumn{2}{|c|}{ Orelha Esquerda } & \multicolumn{2}{|c|}{ Orelha Direita } & \multicolumn{2}{|c|}{ Orelha Esquerda } \\
\hline & $A$ & $B$ & $A$ & $B$ & $A$ & $\mathrm{~B}$ & $A$ & $B$ \\
\hline Mínimo & 33,30 & 53,30 & 46,60 & 33,30 & 46,60 & 33,30 & 20,00 & 13,30 \\
\hline Máximo & 100,00 & 100,00 & 100,00 & 100,00 & 100,00 & 100,00 & 100,00 & 100,00 \\
\hline Média & 82,91 & 86,00 & 80,39 & 88,00 & 81,22 & 81,19 & 78,33 & 72,80 \\
\hline $\begin{array}{l}\text { Desvio } \\
\text { Padrão }\end{array}$ & 19,78 & 12,32 & 19,10 & 16,89 & 16,54 & 19,22 & 20,86 & 20,63 \\
\hline$p$ & \multicolumn{2}{|c|}{0,9346} & \multicolumn{2}{|c|}{0,1601} & \multicolumn{2}{|c|}{0,8075} & \multicolumn{2}{|c|}{0,3459} \\
\hline
\end{tabular}

Não existe diferença significante entre as médias $(p>0,05)$ - Teste de Kruskal-Wallis. 


\begin{tabular}{|l|c|c|c|c|}
\hline \multirow{2}{*}{} & \multicolumn{4}{|c|}{ ALTERAÇÕES NO TESTE PPS } \\
\cline { 2 - 5 } & \multicolumn{2}{|c|}{ GRUPO A } & $\%$ & $\mathrm{c}$ \\
\cline { 2 - 5 } & $\mathrm{N}$ & 6,25 & 0 & $\%$ \\
\hline Orelha Direita murmúrio & 1 & 0,00 & 0 & 0,00 \\
Orelha Direita nomeação & 0 & 6,25 & 0 & 0,00 \\
Orelha Esquerda murmúrio & 1 & 6,25 & 5 & 0,00 \\
Orelha Esquerda nomeação & 1 & 25,00 & 2 & 20,00 \\
2 condições & 4 & 18,75 & 3 & 8,00 \\
3 condições & 3 & 6,25 & 2 & 12,00 \\
4 condições & 1 & 31,25 & 13 & 8,00 \\
Sem alteração & 5 & 100,00 & 25 & 52,00 \\
\hline Total & 16 & & 100,00 \\
\hline
\end{tabular}

Figura 4 - Alterações encontradas no teste PPS, em função do número de indivíduos dos grupos A e B

\section{DISCUSSÃO}

A idade dos avaliados variou de 31 a 51 anos (Figura 1). Isso mostra que todos são adultos, não se classificando como idosos e, portanto, possíveis alterações nas avaliações não podem ser explicadas pelo envelhecimento do sistema auditivo.

Para o tempo de serviço, todos os militares apresentaram variação de 12 a 31 anos (Figura 1).

Diversos autores que pesquisaram a audição de indivíduos expostos a ruído intenso, observaram que as principais mudanças em limiares ou as principais queixas ocorrem até os 12 anos de exposição ao agente agressor 7,9,11,18. Dessa forma, acredita-se que o tempo de exposição a ruído dos militares da presente pesquisa é suficiente para causar danos auditivos. Alguns autores afirmam que a perda da acuidade auditiva se dá principalmente nos primeiros 10 a 15 anos de exposição 5,8,10.

Em relação à idade e ao tempo de serviço dos militares, não se verificou diferença estatisticamente significante entre os grupos A e B (Figura 1). Esse dado é importante, pois mostra que ambos os grupos encontram-se em iguais condições para serem analisados e comparados.

Porém, em relação ao tempo de serviço, este dado discorda de alguns autores que observaram ligação entre a perda auditiva e o tempo de exposição a ruído 6,7,34.

Antes da avaliação do processamento auditivo, objetivo do presente estudo, foi realizada avaliação audiológica básica, pois esta deve sempre preceder a avaliação das funções auditivas centrais ${ }^{23,43}$.

Por meio da audiometria tonal liminar, foi possível fazer a divisão dos militares em dois grupos: grupo A com militares sem perda auditiva (39\%) e grupo $\mathrm{B}$, concatenando militares com perda auditiva $(61 \%)$.
Esse achado concorda com as constatações dos alguns autores que pesquisaram indivíduos expostos a ruído intenso e encontraram percentuais de perda auditiva variando de 50 a $67 \%^{12,16,19}$. A quantidade de alterações observadas no presente estudo é maior que o observado por outros pesquisadores que encontraram percentuais de perda auditiva variando de $21 \%$ a $41 \%$ em trabalhadores expostos a ruído ocupacional ${ }^{11,13,14,20,48}$.

Conhecidamente, a exposição a ruídos de intensidade elevada pode resultar em uma perda auditiva temporária ou permanente ${ }^{4}$. Grande parte das perdas auditivas ocupacionais está ligada à exposição a ruído intenso ${ }^{7}$.

A avaliação do reflexo acústico, bem como a verificação do timpanograma, foram realizadas para descartar qualquer hipótese de alterações de orelha média. Através das medidas de imitância acústica, foi possível comprovar-se a ausência de perdas auditivas de origem condutiva no presente estudo, caracterizando normalidade na orelha média e respeitando os critérios de inclusão dos indivíduos estudados.

Para a avaliação do processamento auditivo, foram empregados os testes de Fala Filtrada, SSW e de Padrão de Freqüência (PPS).

No teste de Fala Filtrada, as médias obtidas pela orelha esquerda foram superiores as obtidas na avaliação da direita, tanto para o grupo $A$, quanto para o $B$ (Tabela 1). Porém, somente para o grupo $B$, tal diferença mostrou-se estatisticamente significante.

Este achado concorda com as afirmações de que no teste de Fala Filtrada, o desempenho da orelha direita costuma ser pior que o da esquerda ${ }^{24}$ e que os resultados obtidos na segunda orelha testada costumam ser melhores que a primeira ${ }^{27}$.

Em estudo semelhante ao presente, também foi verificada diferença estatisticamente signifi- 
cante entre as orelhas direita e esquerda, sendo a esquerda com resultados melhores no teste de Fala Filtrada, tanto para militares com perda auditiva, quanto para aqueles com audição periférica normal ${ }^{34}$

$\mathrm{Na}$ comparação dos resultados do teste de Fala Filtrada, entre os grupos A e B, o grupo de militares com perda auditiva teve pior desempenho tanto para a orelha direita quanto para a esquerda. Somente foi encontrada diferença estatisticamente significante na comparação entre os dois grupos para a orelha direita (Tabela 2).

Em estudo semelhante, tal diferença foi verificada entre dois grupos, um com perda e outro sem perda auditiva, apenas na orelha esquerda ${ }^{34}$.

Em ambos os grupos foram evidenciadas altas porcentagens de alteração no teste de Fala Filtrada: $43,75 \%$ e $68 \%$ dos grupo A e B, respectivamente, obtiveram porcentagens de acerto inferior a $70 \%{ }^{27}$ (Figura 2).

Por mais que as alterações no grupo $B$ sejam maiores que no grupo $A$, ainda assim, considerando-se que no grupo $A$ estão reunidos os militares sem perda de audição, os quase $44 \%$ de alteração configuram uma porcentagem bem elevada.

Estes achados concordam com outros estudos: verificação de porcentagens de alterações no teste de Fala Filtrada de $53 \%$ e $42 \%$ em um grupo exposto apenas a ruído e em um grupo exposto a ruído e a chumbo, respectivamente ${ }^{2}$; com o teste de Fala com Ruído, um teste monótico e de baixa redundância, semelhante ao Fala Filtrada, foi verificado que ele pode ser sensível para identificar alterações auditivas em portadores de PAIR ${ }^{35}$.

Na década de 50 já havia a afirmação de que a audiometria tonal não era suficiente para detectar alterações auditivas centrais e que o teste de Fala Filtrada poderia realizar tal função ${ }^{25}$.

Os achados desta pesquisa discordam de autores que verificaram que o teste de Fala Filtrada não foi capaz de evidenciar as alterações do processamento auditivo em operadores de telemarketing observadas em outros testes ${ }^{44}$. Também discordam de um autor que constatou que, por mais que os monossílabos com significado sejam os mais utilizados na rotina audiológica, eles são de baixa sensibilidade diagnóstica e de alta variabilidade, principalmente quando sensibilizados ${ }^{43}$.

Quanto ao teste SSW (Tabela 3), as médias de erros mostraram-se baixas em todas as condições e não foi observada diferença estatisticamente significante entre os grupos A e B. Tanto nas quatro condições de pesquisa e análise do teste SSW, quanto no total de erros, foi observado poucas alterações: $12,5 \%$ de alterações no grupo $A$ e $4 \%$ no grupo B (Figura 3).
Não foi verificada diferença estatisticamente significante entre os indivíduos com perda e sem perda auditiva, nas condições DNC, DC, EC e ENC do teste SSW. Tal diferença também não foi verificada em outra pesquisa com militares ${ }^{34}$.

O presente estudo concorda com outros autores: a exposição a ruído não influenciou os resultados do teste SSW em português em uma pesquisa ${ }^{33}$; o teste SSW foi nada ou pouco útil para discriminar portadores de PAIR de normo-ouvintes ${ }^{35}$. Além disso, nenhum teste de fala sozinho é sensível para todas as patologias e disfunções do sistema nervoso auditivo central ${ }^{40}$.

Porém, em estudo da função auditiva central de trabalhadores expostos a ruído e solvente, foi concluído que foi possível observar a influência do ruído sobre o processamento auditivo de sons verbais, mesmo com a audição periférica normal, por meio do teste SSW ${ }^{36}$. Para portadores de lesão cerebral, o teste SSW mostrou-se útil na investigação topodiagnóstica ${ }^{32}$.

Percentuais mais altos de alterações no teste SSW foram observados em pesquisa com um grupo de pessoas expostas a ruído intenso e um grupo exposto a ruído e chumbo: 59 e $58 \%$ de alterações, respectivamente. A mesma autora aplicou outra avaliação dicótica, semelhante ao SSW, o teste dicótico de dígitos e encontrou 0\% e 50\% de alterações ${ }^{2}$. O teste dicótico de dígitos também foi aplicado por outros autores para avaliação do processamento auditivo de trabalhadores. Foram observadas $34,4 \%$ e $25 \%$ de alteração em indivíduos expostos a ruído e solventes e em trabalhadores expostos somente a ruído, respectivamente ${ }^{48}$.

O último teste a ser empregado foi o Teste de Padrão de Freqüência (Tabelas 4 e 5, e Figura 4).

Na Tabela 4, não foi observada diferença estatisticamente significante entre a média dos resultados do teste PPS entre as orelhas esquerda e direita, em nenhuma das quatro situações possíveis: resposta murmurada do grupo $A$ e do grupo $B$ e resposta nomeada do grupo A e do grupo B. Outros estudos corroboram estes achados, pois também foi verificado desempenho semelhante para oreIhas direita e esquerda nas condições murmurada e nomeada $26,38,39,54,55$. Não existe vantagem de oreIha em indivíduos normais nos testes de ordem e seqüência temporal ${ }^{37}$.

Em relação comparação entre o desempenho dos indivíduos dos grupos A e B para as quatro situações testadas por meio do PPS (murmurado OD e OE, nomeado OD e OE), não foi verificada diferença estatisticamente significante. Isso mostra que os resultados obtidos pelo grupo com perda auditiva e sem perda auditiva foram semelhantes (Tabela 5). 
Estes achados concordam com um trabalho em que também não foi encontrada diferença estatisticamente significante ao ser feita a comparação entre dois grupos de militares expostos a ruído intenso: um com e outro sem perda de audição ${ }^{34}$.

$\mathrm{Na}$ Figura 4, foram demonstradas as alterações observadas no teste PPS. Foi totalizado $68,75 \%$ de alterações em uma, duas, três ou nas quatro condições de pesquisa para o grupo $A$. Os militares do grupo B apresentaram um percentual mais baixo, de $48 \%$ de resultados abaixo do normal.

Sabe-se que testes como o PPS não são influenciados por perdas auditivas periféricas ${ }^{26,38,39,55}$. Os achados desse estudo concordam, então, com esta afirmação, visto que as alterações foram encontradas em ambos os grupos, sendo, inclusive, maiores no grupo sem perda auditiva.

Estes achados também concordam com uma pesquisa do processamento auditivo de 76 trabaIhadores de uma gráfica, na qual foram encontrados resultados de $25 \%$ e $33,4 \%$ de alteração no teste PPS em indivíduos expostos a ruído e solventes e em trabalhadores expostos somente a ruído, respectivamente ${ }^{48}$.

Observa-se, então, que houve um grande número de alterações nos testes de Fala Filtrada e PPS, não observadas em grande número no teste SSW.

É sabido que, devido à complexidade do sistema nervoso auditivo central, os efeitos de uma determinada lesão podem ser tão sutis que ela não será detectada por um teste, mas claramente visível por outro. Os efeitos dos distúrbios do sistema nervoso auditivo central, em geral, são completamente discretos e os resultados dos testes altamente variáveis ${ }^{42}$.

Foram verificadas alterações de processamento auditivo em ambos os grupos. Já havia sido concluído em outro estudo com militares que a exposição a elevados níveis de pressão sonora tem efeitos deletérios no processamento auditivo de militares, independente de haver alteração auditiva periférica ${ }^{34}$.

A alteração ocorrida no processamento auditivo, causada por exposição a ruído intenso também foi confirmada em diversas pesquisas na literatura audiológica ${ }^{2,42,44-48}$.

\section{CONCLUSÃO}

$\mathrm{Na}$ avaliação do processamento auditivo, observou-se, principalmente que em praticamente todas as situações de avaliação, não houve diferença estatisticamente significante entre os grupos A e B, apesar do grande número de alterações no teste de Fala Filtrada e PPS. Isso mostra que, possivelmente, as alterações auditivas centrais ocorrem antes mesmo de haver perda auditiva periférica.

Dessa forma, conclui-se que a exposição a ruído ocupacional interfere no processamento auditivo de militares.

\begin{abstract}
Purpose: to evaluate the auditory processing of military personnel exposed to occupational noise. Methods: 41 servicemen, exposed to noise for at least 10 years were evaluated, divided into Group $A$ $(n=16)$, without hearing loss and Group $B(n=25)$, with hearing loss. The following evaluations were carried through: basic audilogic evaluation and auditory processing tests (low-filtered, SSW and Pitch Pattern Sequence tests). Results: there were high incidences of auditory processing alterations, especially at low-filtered test (43.75\% and $68 \%$ on groups A e B, respectively) and Pitch Pattern Sequence test $(68.75 \%$ and $48 \%$, on groups A e B, respectively). The SSW test was not efficient to evaluate the central hearing abilities of people exposed to high levels of sound pressure. Conclusion: the occupational noise exposure interferes in the auditory processing of military personnel. The alterations on central auditory pathways can be verified even if there is no any alteration on peripheral hearing.
\end{abstract}

KEYWORDS: Hearing; Noise, Occupational; Hearing Tests

\section{REFERÊNCIAS}

1. Swilinska-Kowalska M. Hearing loss related to exposures to noise and chemicals. In: $22^{\circ}$ Encontro Internacional de Audiologia (EIA); 2007, Mar 28 - 31; Natal, Rio Grande do Norte. CD-ROM.

2. Jacob LCB. Efeitos da exposição simultânea ao chumbo e ao ruído sobre o sistema nervoso auditivo central em trabalhadores de uma fábrica de baterias [doutorado]. São Paulo (SP): Universidade Federal de São Paulo; 2000.

3. Habermann J. Ueber die schwerhörigekeit der kesselschmiede. Archiv für Ohrenheilkunde. 1890; 30:1-25. 
4. Jerger S, Jerger J. Alterações auditivas. Rio de Janeiro: Atheneu; 1989. 204 p.

5. Comitê Nacional de Ruído e Conservação Auditiva. Perda auditiva induzida pelo ruído relacionada ao trabalho. Acta Awho. 1994; 13(3):126-7.

6. Melnick W. Saúde auditiva do trabalhador. In: Katz J. Tratado de audiologia clínica. São Paulo: Manole; 1999. p. 529-47.

7. Morata TC, Lemasters GK. Considerações epidemiológicas para o estudo das perdas auditivas ocupacionais. In: Nudelmann AA, Costa EA, Seligman J, Ibañez RN. Perda auditiva induzida pelo ruído. v.

2. Rio de Janeiro: Revinter; 2001. p. 1-16.

8. Brasil. Ministério da Saúde. Secretaria de Atenção à Saúde. Perda auditiva induzida por ruído (Pair). Brasília: Ministério da Saúde; 2006.

9. Glorig A, Ward WD, Nixon J. Damage risk criteria and noise-induced hearing loss. Arch Otolaryngol. 1961; 74:413-23.

10. Taylor W, Pearson J, Mair A, Burns W. Study of noise and hearing in jute weaving. J Acoust Soc Am. 1965; 38:113-20.

11. Quick TC, Lapertosa JB. Contribuição ao estudo das alterações auditivas e de ordem neuro-vegetativas atribuíveis ao ruído. Rev Bras Saúde Ocup. 1981; 9(36):50-6.

12. Talbott EO, Findlay RC, Kuller LH, Lenkner LA, Matthews KA, et al. Noise-induced hearing loss: a possible marker for high blood pressure in older noise-exposed population. J Occup Med. 1990; 32(8):690-7.

13. Araújo SA. Perda auditiva induzida pelo ruído em trabalhadores de metalúrgica. Rev Bras Otorrinolaringol. 2002; 68(1):47-52.

14. Gonçalves CGO, Iguti AM. Análise de programas de preservação da audição em quatro indústrias metalúrgicas de Piracicaba, São Paulo, Brasil. Cad Saúde Públic. 2006; 22(3):609-18.

15. Fiorini AC, Silva AS, Bevilacqua MC. Ruído, comunicação e outras alterações. Rev Saúde Ocup Segur. 1991; 26:49-60.

16. Santos CCS. Avaliação otoneurológica em indivíduos expostos a ruído ocupacional [monografia]. Santa Maria (RS): Universidade Federal de Santa Maria; 2003.

17. Guida HL. Efeitos psicossociais da perda auditiva induzida pelo ruído em ex-funcionários da indústria. Acta Otorrinolaringol. 2007; 25(1):78-83.

18. Axelsson A, Hamernik RP. Acute acoustic trauma. Acta Otolaryngol. 1987; 104(3-4):225-33.

19. Ylikoski ME, Ylikoski JS. Hearing loss and handicap of professional soldiers exposed to gunfire noise. Scand J Work Environ Health. 1994; 20(2):93-100.

20. Silva AP, Costa EA, Rodrigues SMM, Souza HLR, Massafera VG. Avaliação do perfil auditivo de militares de um quartel do Exército Brasileiro. Rev Bras Otorrinolaringol. 2004; 70(3):344-50.

21. Brasil. Portaria $n^{\circ} 24$ de 29/12/1994 - NR-7. Programa de Controle Médico de Saúde Ocupacional. (D.O.U.30/12/1994).

22. Schochat E. Percepção de fala em perdas auditivas neurossensoriais. In: Lichtig I, Carvallo RMM. Audição: abordagens atuais. Carapicuíba: PróFono; 1997. p. 223-35.

23. Alvarez AMMA, Balen SA, Misorelli MIL, Sanchez ML. Processamento auditivo central: proposta de avaliação e diagnóstico diferencial. In: Caovilla HH, Ganança MM, Munhoz MSL, Garcia ML. Audiologia clínica. São Paulo: Atheneu; 2000. p. 120-30.

24. Bellis TJ. Assessment and management of central auditory processing disorders: from science to practice. San Diego: Singular; 1996.

25. Bocca E, Calearo C, Cassinari V. A new method for testing hearing in temporal lobe tumors; preliminary report. Acta Otolaryngol. 1954; 44(3):219-21.

26. Sanchez ML. Avaliação do processamento auditivo em idosos que relatam ouvir bem [mestrado]. São Paulo (SP): Universidade Federal de São Paulo; 2002.

27. Pereira LD, Schochat E. Baixa redundância: fala filtrada e fusão biaural. In: Pereira LD, Schochat E. Processamento auditivo central: manual de avaliação. São Paulo: Lovise; 1997. p. 103-9.

28. Katz J. The use of SSW for assessing the integrity of central auditory nervous system. J Audit Res. 1962; 2:227-37.

29. Borges ACLC. Adaptação do teste SSW para a língua portuguesa: nota preliminar. Acta Awho. 1986; 5(suppl. 1):38-40.

30. Borges ACLC. Dissílabos alternados - SSW. In: Pereira LD, Schochat E. Processamento auditivo central: manual de avaliação. São Paulo: Lovise; 1997. p. 169-78.

31. Quintero SM, Marotta RMB, Marone SAM. Limites para o teste de reconhecimento de dissílabos em tarefa dicótica (SSW) em indivíduos idosos. Arq Int Otorrinolaringol. 2002; 6(3):216-23.

32. Teixeira RO. Diagnóstico topográfico neurológico: neuroimagem $\mathrm{X}$ teste dicótico de dissílabos alternados - SSW [mestrado]. Santa Maria (RS): Universidade Federal de Santa Maria; 2002.

33. Gil D, Borges ACC, Pereira LD. Teste de escuta dicótica de dissílabos em indivíduos portadores de deficiência auditiva neurossensorial, com e sem exposição a ruído. Acta Awho. 1999; 18(1):12-7.

34. Marins KKRC. Do processamento auditivo em militares expostos a elevados níveis de pressão sonora [mestrado]. Santa Maria (RS): Universidade Federal de Santa Maria; 2004. 
35. Moreira RR, Ferreira Junior M. Testes de fala: aplicação em portadores de perda auditiva induzida por ruído. Pró-Fono. 2004; 16(3):293-300.

36. Toniolo IMF. Função auditiva central: caracterização de respostas através de tarefa dicótica em trabalhadores expostos a ruído e solvente [mestrado]. Santa Maria (RS): Universidade Federal de São Paulo; 1999.

37. Balen SA. Processamento auditivo central: aspectos temporais da audição e percepção acústica da fala [mestrado]. São Paulo (SP): Pontifícia Universidade Católica de São Paulo; 1997.

38. Pinheiro ML, Musiek FE. Sequencing and temporal ordering in the auditory system. In: Pinheiro $\mathrm{ML}$, Musiek FE. Assessment of central auditory dysfunction: foundations and clinical correlates. Baltimore: Williams \& Wilkins; 1985. p. 219-38.

39. Musiek FE, Pinheiro ML. Frequency patterns in cochlear, brainstem, and cerebral lesions. Audiology. 1987; 26(2):79-88.

40. Mueller HG, Bright KE. Testes centrais: procedimentos utilizando monossílabos. In: Katz J. Tratado de audiologia clínica. São Paulo: Manole; 1999. p. 220-36.

41. Schoeny ZG, Talbott RE. Testes centrais: procedimentos utilizando estímulos não-verbais. In: Katz J. Tratado de audiologia clínica. São Paulo: Manole; 1999. p. 210-9.

42. Musiek FE, Lamb L. Avaliação auditiva central: uma visão geral. In: Katz J. Tratado de audiologia clínica. São Paulo: Manole; 1999. p. 195-209.

43. Costa EA. Um teste de fala, com ruído competidor, para aplicação em audiologia ocupacional. In: Nudelmann AA, Costa EA, Seligman J, Ibañez RN. Perda auditiva induzida pelo ruído. v. 2. Rio de Janeiro: Revinter; 2001. p. 62-77.

44. Silva MCB, Cunha MB, Souza CCL, Mitre El. Avaliação do processamento auditivo em operadores de telemarketing. Rev CEFAC. 2006; 8(4):536-42.

45. Saunders JC, Dear SP, Schneider ME. The anatomical consequences of acoustic injury: a review and tutorial. J Acoust Soc Am. 1985; 78(3):833-60.
46. Santos UP, Morata TC. Efeitos do ruído na audição. In: Santos UP, organizador. Ruído: riscos e prevenção. São Paulo: Hucitec; 1996. p. 43-53.

47. Bernardi APA. Trabalhadores expostos simultaneamente a ruído e tolueno: estudo das emissões otoacústicas evocadas transitórias e efeito de supressão [mestrado]. São Paulo (SP): Faculdade de Saúde Pública da Universidade de São Paulo; 2000.

48. Santoni CB, Drobina EF, Misorelli MI. Avaliação do processamento auditivo em trabalhadores de uma indústria gráfica. In: Bernardi APA, organizador. Conhecimentos essenciais para atuar bem em empresas: audiologia ocupacional. São José dos Campos: Pulso; 2003. p. 81-90.

49. ISO 1999. Acoustics: determination of occupational noise exposure and estimation of noise-induced hearing impairmant. 2. ed. Genève; 1990.

50. Jerger J. Clinical experience with impedance audiometry. Arch Otolaryngol. 1970; 92(4):311-24.

51. Pereira LD. Processamento auditivo central: abordagem passo a passo. In: Pereira LD, Schochat E. Processamento auditivo central: manual de avaliação. São Paulo: Lovise; 1997. p. 49-59.

52. Chaves AD. Uma nova proposta para avaliação do reconhecimento da fala em adultos com audição normal [mestrado]. Santa Maria (RS): Universidade Federal de Santa Maria; 1997.

53. Pillon L. Análise da percepção da fala em crianças com audição normal: uma nova proposta [mestrado]. Santa Maria (RS): Universidade Federal de Santa Maria; 1998.

54. Auditec. Evaluation manual of pitch pattern sequence and duration pattern sequence. Missouri; 1997.

55. Corazza MCA. Avaliação do processamento auditivo central em adultos: teste de padrões tonais auditivos de freqüência e teste de padrões tonais auditivos de duração [doutorado]. São Paulo (SP): Universidade Federal de São Paulo; 1998.

RECEBIDO EM: 10/06/2007

ACEITO EM: 04/02/2008

Endereço para correspondência:

Rua Benjamin Constant, 1198, ap. 303

Santa Maria - RS

CEP: $97050-022$

Tel: (55) 30284591

E-mail: santos.carlas@gmail.com 